\title{
ASSOCIAÇÃO ENTRE RAÇA E INCIDÊNCIA DE DOENÇA RENAL TERMINAL SECUNDÁRIA A GLOMERULONEFRITE: INFLUÊNCIA DO TIPO HISTOLÓGICO E DA PRESENÇA DE HIPERTENSÃO ARTERIAL
}

*A.A. Lopes, M.A. Silveira, R.P. Martinelul, H. Rocha

Trabalhorealizadono Hospital Universitário Prof. EdgardSantos, UniversidadeFederal daBahia, Salvador, Ba

RESUMO - ОвJetivo. Avaliar retrospectivamente a associação entre raça e incidência de doença renal terminal (DRT) em pacientes com glomerulonefrites em um Hospital Universitário do estado da Bahia.

Métodos. A amostra foi composta de 79 pacientes com esclerose glomerular focal (EGF), 50 com glomerulonefrite membranoproliferativa (GNMP) e 44 com outros tipos de glomerulonefrites (OTGN), acompanhados entre 1970 e 1996 por pelo menos 6 meses. Não foram incluídos os pacientes com glomerulonefrite aguda, formas crescênticas, lesão mínima, doenças do colágeno e com níveis de creatinina sérica maior ou igual a $4 \mathrm{mg} / \mathrm{dl}$. Quanto à raça, os pacientes foram classificados em brancos, mulatos ou negros. Para idades superiores a 18 anos e na ausência de uso de anti-hipertensivos, definiu-se como hipertensos pacientes que tiveram médias das três primeiras determinações da pressão arterial sistólica ou diastólica iguais ou superiores a $140 \mathrm{e}$ $90 \mathrm{mmHg}$, respectivamente; para idades igual ou abaixo de 18 anos foram utilizados critérios recomendados pela "Task Force on Blood Pressure in Children".
Resultados. No grupo de normotensos, a incidência de DRT foi $72 \%$ menor em mulatos e negros (risco relativo $(R R)=0,28$; intervalo de confiança (IC) $95 \%=0, I I-0,67$ ). No grupo de hipertensos, a associação entre raça e DRT apresentou direção inversa $(R R=I, 49 ;$ IC $95 \%=0,68-4,34 ; p=0,316)$ à observada em normotensos. Esta variação no $R R$ de acordo com a presença ou ausência de HA foi estatisticamente significante $(p=0,0 \mathrm{l})$. Entre os normotensos, parte da diferença racial na incidência de DRT pode ser explicada pela distribuição de tipos histológicos de glomerulonefrite entre o grupo de brancos e o grupo de negros e mulatos.

CONCLUSÖEs. Entre os normotensos com glomerulonefrite, a incidência de DRT foi significantemente maior em brancos do que em negros ou mulatos. Contrariamente, entre os hipertensos observou-se uma tendência para um risco maior de DRT no grupo de negros e mulatos. $\mathrm{O}$ estudo sugere que $\mathrm{HA}$ e $\mathrm{O}$ tipo histológico de glomerulonefrite influenciam a associação entre raça e DRT.

Unitermos: Raça. Negro. Doença Renal Terminal. Glomerulonefrite.

\section{INTRODUÇÃO}

Glomerulonefrite continua sendo uma importante causa de doença renal terminal (DRT)'. Nos Estados Unidos, a incidência de DRT atribuída às glomerulonefrites primárias é aproximadamente duas vezes maior nos negros do que nos brancos ${ }^{21}$. As razões para estas diferenças raciais, entretanto, não são claras. Apesar da evidência de que o negro hipertenso apresenta uma

*Correspondência R. Mal. Floriano, 448/1301 Cep: 401 10-010-Salvador-BA E-mail:aaslopes@utba.br redução mais rápida da função renal do que o branco hipertenso ${ }^{32}$, não tem sido avaliado se a associação raça versus DRT em pacientes com glomerulonefrites difere entre grupos de pessoas com e sem hipertensão arterial (HA). É também interessante explorar uma possível influência da distribuiçãa dos tipos histológicos de glomerulonefrites nesta associação (i.e., entre raça e vs DRT), considerando as diferenças regionais ${ }^{4-6}$ e raciais ${ }^{7,8}$ na distribuição das glomerulonefrites. $O$ presente trabalho estuda uma amostra de pacientes portadores de glomerulonefrites residentes no estado da
Bahia, com o objetivo básico de avaliar se raça está associada com a incidência de DRT. São também avaliadas as influências do tipo histológico e da presença de hipertensão arterial na relação entre raça e DRT.

\section{Métodos e Casuística}

Foram estudados, retrospectivamente, 173 pacientes (média de idade $=23,7 \pm$ 13,9 anos; mediana $=22,3$ anos) com o diagnóstico histológico de glomerulonefrite, acompanhados no Hospital Universitário Prof. Edgard Santos da Universidade Federal da Bahia (HUPES), entre 1970 e 
Raça e Doença Renal Terminal

1996. Neste período, em torno de 966 pacientes tiveram laudos de biopsias renais arquivados no Serviço de Anatomia Patológica do HUPES. Foram incluídos no presente estudo os pacientes com pelo menos 6 meses de acompanhamento (mediana de acompanhamento $=47,6$ meses) e com níveis iniciais de creatinina sérica inferior a 4,0 mg/dl. Foram excluídos os pacientes portadores de glomerulonefrite aguda, formas crescênticas, lesão mínima ou doenças do colágeno. O tempo de seguimento foi definido como o intervalo entre a primeira consulta e o diagnóstico de DRT (ou última consulta na ausência de DRT). Definiu-se como DRT o momento em se tornou necessário o início de terapia de substituição da função renal. Foram definidos três grupos de acordo com o tipo histológico: GNMP, $n=50$; EGF, $n=79$; e outras glomerulonefrites (OTGN), $n=44$. O grupo OTGN foi formado por 4 I pacientes com formas proliferativas não classificadas como GNMP e três pacientes com glomerulonefrite membranosa. Os pacientes foram inicialmente classificados quanto à raça em brancos $(n=53)$, mulatos $(n=108)$ e negros $(n=12)$, de acordo com o prontuário médico. A classificação racial utilizada desde a década de 1960 no HUPES segue critérios publicados por Krieger et $a$. . . Por razões estatísticas (pequeno número de negros), a variável raça foi tratada como dicotômica, agrupando mulatos e negros em uma única categoria. Idade ( $\leq 18$ e $>18$ anos), sexo, $H A$, tipo histológico (GNMP $(n=50), E G F$ $(n=80)$ e OTGN $(n=49))$ foram tratados como potenciais confundidoras ou modificadoras de efeito da associação entre raça e incidência de DRT. Pacientes usando drogas anti-hipertensivas, excluindo diuréticos na vigência de síndrome nefrótica, foram classificados como hipertensos. Na ausência de anti-hipertensivos, pacientes com idade $>18$ anos foram considerados hipertensos se apresentassem uma média ${ }^{3} \mid 40$ mm Hg nas três primeiras medidas da pressão arterial sistólica ou 390 mm Hg nas medidas da pressão arterial diastólica. Para indivíduos com idades $\leq 18$ anos foram usados os critérios recomendados pela Task Force on Blood Pressure in Children ${ }^{510}$.

Análise Estatística. Em todas as comparações raciais, negros e mulatos foram incluídos na mesma categoria; o grupo de pacientes o grupo dos brancos foi utilizado como referência. Idade ( $\leq 18$ e $>18$ anos), sexo, HA e tipo histológico foram tratados como potenciais confundidores ou modificadores de efeito da associação entre raça e incidência de DRT. Nas análises tabulares, a medida de freqüência de DRT foi densidade de incidência" ". O tempo de seguimento foi definido como o intervalo entre a data da primeira consulta e data do diagnóstico de DRT (ou data da última consulta em pacientes que não desenvolveram DRT).

Nessas análises, o efeito modificador de co-variáveis sobre a associação entre raça e incidência de DRT foi avaliado através de um teste de homogeneidade utilizando o módulo RATES2 do "Computer Programs for Epidemiologic Analysis", versão 3 $(\text { PEPI })^{12}$. Através do mesmo módulo estimou-se o risco relativo (RR) ajustado pela técnica de Mantel-Haenszel e o respectivo intervalo de confiança $(\mathrm{IC})$ através de um método exato (mid-p exact). Comparações de médias e de proporções foram feitas através do teste t e do teste do qui-quadrado, respectivamente, utilizando-se procedimentos do "Statistical Package for the Social Sciences", SPSS '13. Utilizou-se o mesmo software para testes de medianas.

Ajustes multivariados da associação entre raça e DRT foram feitos através de modelos de risco proporcional (Cox proportional hazard models ou modelos de Cox $)^{14}$. Nesses modelos de risco proporcional, os eventos dicotômicos (ex. DRT) foram categorizados como I, indicando ocorrência, e 0 (zero), indicando não ocor- rência; as variáveis preditoras dicotômicas (ex. raça: grupo de negros e mulatos vs grupo de brancos) foram codificadas como I, indicando grupo de exposição (ex., grupo de negros e mulatos), e 0 (zero), indicando grupo de referência (ex., grupo de brancos). Para avaliar a influência do tipo histológico (GNMP, EGF e OTGN), foram definidas duas variáveis indicadoras (dummy variables), utilizando-se EGF como grupo de referência. Nos modelos de Cox, a presença de modificação de efeito (ou interação estatística) foi avaliada através do coeficiente do produto entre duas co-variáveis. Como raça foi a variável independente (preditora) de maior interesse, testaramse as interações envolvendo esta variável e cada uma das co-variáveis, utilizando-se DRT como variável dependente (evento). Ficou definido que na presença de interação estatisticamente significante seriam utilizados modelos específicos para cada nível da variável modificadora de efeito (análise estratificada). A premissa do modelo proporcional de Cox de que o RR deve ser constante ao longo do tempo foi avaliada através do paralelismo das curvas de sobrevida (log minus log plot). Os testes estatísticos foram bi-caudais, sendo considerados significantes valores de p menores do que 0,05 (5\%). Foram considerados significantes valores de p menores do que 5\%, para testes estatísticos bi-caudais

\section{Resultados}

Na tabela I, as características dos pacientes são descritas de acordo com os grupos raciais: mulato + negro versus branco. A média (23, I \pm I3,6 versus $25,0 \pm 14,7$ anos) e mediana ( 21,5 versus 23,0 anos) de idade foram discretamente menores no grupo de mulatos e negros. Em concordância com estes achados, a freqüência de indivíduos com idade $>18$ anos foi um pouco menor no grupos dos negros e mu- 
latos (60\%) do que no grupo dos (63\%) brancos. Houve um predomínio de pessoas do sexo masculino na amostra; $60 \%$ do grupo dos mulatos e negros e $62,3 \%$ do grupo dos brancos. A prevalência de HA na fase inicial do acompanhamento foi apenas um pouco maior nos brancos (44,4 vs $40,8 \% ; p=0,65)$. Nenhuma dessas diferenças raciais alcançou significância estatística $(p>0,1)$. Por outro lado, observou-se uma associação estatisticamente significante $(p=0,004)$ entre raça e tipo histológico. GNMP foi o tipo histológico mais freqüente em brancos e EGF o tipo mais prevalente em negros e mulatos.

A tabela 2 mostra que a incidência de DRT foi 4 I\% menor (RR=0,59; IC 95\% $=0,31-1,12 ; p=0,107)$, embora estatisticamente não significante, no grupo dos negros e mulatos (incidência $=25,4 / 10^{4}$ pessoas-mês) do que no grupo dos brancos (incidência $=43,3 / 10^{4}$ pessoas-mês). Quando a associação entre raça e DRT foi ajustada para a idade pela técnica de MantelHaenszel, o RR praticamente não se alterou $\left(R R_{M H}=0,58 ;\right.$ IC $\left.95 \%=0,31-1,12 ; p=0,105\right)$. As estimativas pontuais dos RRs de raça foram 0,73 (IC 95\%=0,20-2,92) no grupo etário $\leq 18$ anos e 0,54 (IC 95\%=0,24$1,23)$ no grupo etário $>18$ anos. Esses RRs não foram significantemente diferentes de acordo com o teste de homogeneidade $(p=0,690)$.

Também após o ajuste para $\mathrm{HA}$, o RR da associação entre raça e DRT permaneceu menor do que I $\left(\mathrm{RR}_{\mathrm{MH}}=0,63 ; \mathrm{IC} 95 \%=\right.$ $0,32-1,19)$, indicando um menor risco de DRT nos negros e mulatos. Foi observado, no entanto, um importante e estatisticamente significante $(p=0,01)$ efeito modificador de HA na associação entre raça e incidência de DRT. Entre os pacientes normotensos, a incidência de DRT no grupo dos negros e mulatos foi aproximadamente $72 \%$ menor do que no grupo dos brancos (RR=0,28; IC 95\%=0, I I-0,67).

Tabela I - Características demográficas e duração do acompanhamento dos pacientes nos grupos raciais.

\begin{tabular}{|c|c|c|c|}
\hline & \multicolumn{2}{|c|}{ Grupos Raciais } & \multirow[b]{2}{*}{$\begin{array}{l}\text { Valor } \\
\text { de p } \\
0,410 \\
0,298 \\
0,605\end{array}$} \\
\hline $\begin{array}{l}\text { média } \pm \text { DP } \\
\text { mediana } \\
\% \text { idade }>18 \text { anos (n/N) }\end{array}$ & $\begin{array}{c}\text { Mulato+Negro } \\
N=120 \\
23,1 \pm 13,6 \\
21,5 \\
60,0(72 / 120)\end{array}$ & $\begin{array}{c}\text { Branco } \\
N=53 \\
25,0 \pm 14,7 \\
23,0 \\
64,2(34 / 53)\end{array}$ & \\
\hline Sexo $\%$ Masculino (n/N) & $60,0(72 / 120)$ & $62,3(33 / 53)$ & 0,779 \\
\hline $\begin{array}{l}\text { Tipo Histológico } \\
\% \text { GNMP (n/N) } \\
\% \text { EGF (nNN) } \\
\% \text { OTGN (n/N) }\end{array}$ & $\begin{array}{l}21,7(26 / / 20) \\
48,3(58 / 120) \\
30,3(36 / 120)\end{array}$ & $\begin{array}{l}45,3(24 / 53) \\
39,6(21 / 53) \\
15,1(08 / 53)\end{array}$ & 0,004 \\
\hline $\begin{array}{l}\text { Hipertensão Arterial } \\
\text { \%Hipertensos (n/N) }\end{array}$ & $40,0(48 / / 20)$ & $45,3(24 / 53)$ & 0,516 \\
\hline
\end{tabular}

Tabela 2 - Comparação das incidências de doenças renal terminal entre os grupos raciais (negro+mulato vs branco)

\section{Associação não ajustada}

Número de pacientes

$\begin{array}{lccccc}\text { Raça } & \text { com }^{\uparrow} \text { DRT } & \text { Pacientes-mês } & \text { pacientes-mês } & \text { RR(IC 95\%) } & \text { valor de } p \\ \text { Negro } & 21 & 8274 & 25,4 & 0,59(0,31-1,12) & 0,107\end{array}$

+mulato

$\begin{array}{lllll}\text { Branco } & 17 & 3923 & 43,3 & \text { Referência }\end{array}$

Teste de Homogeneidade para o risco relativo (RR) e ajuste para idade Idade

$\begin{array}{ccc}\leq 18 \text { anos } & >\text { I8anos } & \text { Teste de } \\ & & \text { Homogeneidade } \\ \text { RR(IC 95\%) } & \text { RR(IC 95\%) } & p=0,690 \\ 0,73(0,20-2,92) & 0,54(0,24-1,23) & \\ p=0,621 & p=0,1 \mid 1 & \end{array}$

Ajuste pela técnica de Mantel-Haenszel (MH)

Teste de Homogeneidade para o risco relativo (RR) e ajuste para hipertensão arterial Hipertensão Arterial

\begin{tabular}{|c|c|c|c|c|}
\hline \multicolumn{2}{|c|}{ Hipertensão Arterial } & \multirow{3}{*}{$\begin{array}{c}\text { Teste de } \\
\text { Homogeneidade } \\
p=0,010\end{array}$} & \multirow{2}{*}{\multicolumn{2}{|c|}{$\begin{array}{l}\text { Ajuste pela técnica de } \\
\text { Mantel-Haenszel (MH) }\end{array}$}} \\
\hline & & & & \\
\hline $\begin{array}{c}\operatorname{RR}(I C 95 \%) \\
0,28(0,1 \mid-0,67) \\
D=0,005\end{array}$ & $\begin{array}{c}\operatorname{RR}(\operatorname{IC} 95 \%) \\
I, 49(0,56-4,30) \\
D=0,439\end{array}$ & & $\begin{array}{l}\operatorname{RR}_{\text {MH }}(\operatorname{IC} 95 \%) \\
0,63(0,32-1,19)\end{array}$ & $\begin{array}{l}\text { valor de } p \\
0,144\end{array}$ \\
\hline
\end{tabular}

\section{${ }^{\uparrow}$ Doença Renal Terminal}

${ }^{*} \mathrm{RR}_{\mathrm{MH}}=$ Risco Relativo ajustado pela técnica de Mantel-Haenszel

Essa associação entre raça e DRT no grupo de normotensos foi estatisticamente significante $(p=0,005)$. Contrariamente, no grupo dos hipertensos houve uma tendên- cia para inversão da associação $(R R=1,49$; IC 95\%=0,56-4,30) quando comparada com a observada em normotensos, sugerindo um maior risco de DRT em negros e 


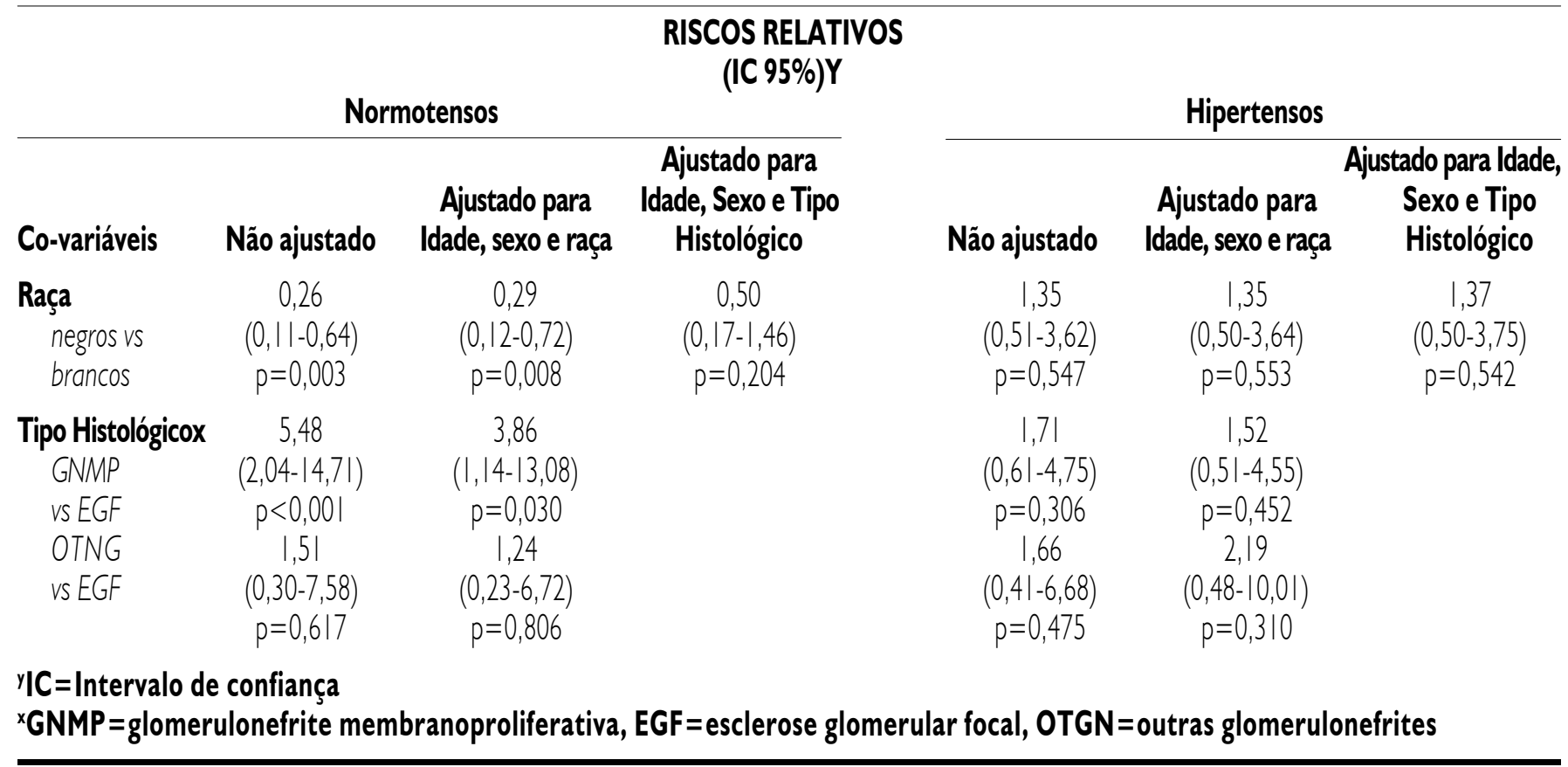

mulatos do que em brancos.

Nos modelos de risco proporcional (regressão de Cox) os coeficientes de interação raça*idade, raça*sexo e raça*tipo histológico foram estatisticamente não significantes $(p>0,1)$. Concordante com 0 teste de homogeneidade pela técnica de Mantel-Haenszel, o coeficiente de interação raça*HA na regressão de Cox foi estatisticamente significante $(p<0,0 \mid 2)$. Por esta razão foram utilizados modelos específicos para hipertensos e para normotensos. Em concordância com as comparações entre densidades de incidências mostradas na tabela 2, na análise não ajustada através do modelo de risco proporcional, a incidência de DRT no grupo de normotensos foi significantemente maior nos brancos ( $R R=$ 0,26; IC 95\%=0, I - -0,64; $p=0,003)$. Após o ajuste para a idade e sexo, a associação entre raça e incidência de DRT praticamente não se alterou e se manteve estatisticamente significante $(R R=0,29 ;$ IC 95\%= 0, I2-0,72; $p=0,008)$. Após a inclusão do tipo histológico no modelo de Cox, no entanto, o RR se aproximou da hipótese nula e a associação entre raça e DRT se tornou estatisticamente não significante (RR ajustado=0,50; IC 95\%=0, I7-I,46; $p=$ 0,204). Também em concordância com a análise tabular (tabela 2) no modelo de Cox, específico para hipertensos, a associação entre raça e DRT (tabela 3) apresentou direção contrária ao observado no grupo de normotensos. No grupo de hipertensos, as associações entre raça e DRT - não ajustada $(R R=1,35)$, ajustada para sexo e idade $(R R=1,35)$ e a ajustada para todas as covariáveis $(R R=1,37)$ - foram similares e estatisticamente não significantes $(p>0, I)$.

A tabela 3 também mostra as estimativas da associação entre tipo histológico e DRT através do modelo de Cox; EGF foi utilizada como grupo de referência. No grupo de normotensos, a incidência de DRT foi mais de cinco vezes maior em pacientes com GNMP do que naqueles com EGF (RR=5,48; IC 95\%=2,04-|4,7|; $p<0,001$ ). Após 0 ajuste para a idade, sexo e raça a associação tornou-se mais fraca (RR ajustado=3,86; IC
$95 \%=1,14-13,08)$, permanecendo, no entanto, estatisticamente significante. Os RRs da associação OTGN vs EGF foram estatisticamente não significantes e menores do que os RRs da associação entre OTGN vs EGF; RR (ORGN vs EGF) não ajustado $=\mid, 51$ (IC 95\%=0,30-7,58; $p=0,617)$ e o ajustado = I,24 (IC 95\%=0,23-6,72; $p=0,806)$.

No grupo de hipertensos, a incidência de DRT foi também maior em pacientes com GNMP do que em pacientes com EGF, sendo esta associação, no entanto, mais fraca do que a observada no grupo de normotensos: RR não ajustado $=1,7 \mathrm{I}$ (IC $95 \%=0,61-4,75)$ e RR ajustado $=1,52$ (IC $95 \%=0,51-4,55)$. Similarmente ao observado em normotensos, a incidência de DRT foi maior em pacientes com OTGN do que em pacientes com EGF; RR (OTGNvs EGF) não ajustado $=1,66$ (IC 95\%=0,4l-6,68) e o ajustado $=2,19$ (IC 95\%=0,4810,0 I). Nenhuma das estimativas da associação tipo histológico vs DRT no grupo de hipertensos, no entanto, alcançou significância estatística $(p>0,1)$. 


\section{Discussão}

Os dados do presente estudo sugerem que, entre pacientes do estado da Bahia com glomerulonefrites primárias, os negros e mulatos apresentam uma menor incidência de DRT do que os brancos. De acordo com os resultados esta associação entre raça e DRT é independente da idade. É importante destacar que antes de implementar o estudo previa-se que a incidência de DRT seria maior em negros e mulatos. Nossos dados, no entanto, não apóíam a hipótese de que mulatos e negros com glomerulonefrites residentes no estado da Bahia apresentem um maior risco de DRT do que os brancos. Embora o tamanho da amostra tenha sido um fator limitante para se rejeitar a hipótese nula de que os riscos de DRT são iguais entre o grupo de brancos e o constituído de negros e mulatos, é importante observar que, em verdade, a associação entre raça e DRT apresentou direção contrária à expectativa, visto que a incidência de DRT foi maior nos brancos do que nos negros e mulatos. Essas diferenças raciais no grupo de normotensos permaneceram estatisticamente significantes, mesmo após o ajuste simultâneo para as variáveis idade e sexo.

A expectativa de que o risco de DRT seria maior em negros e mulatos foi, em parte, influenciada por observações estudos anteriores, indicando uma redução mais rápida da função renal, ao longo dos anos, em pessoas negras do que em brancas, mesmo na ausência de HA e de diabetes mellitus ${ }^{15}$. Além do mais, uma maior susceptibilidade para $H A$, particularmente formas mais graves ${ }^{11,12}$, e para lesão renal secundária a HA tem sido observada em negros ${ }^{2,13}$.

A hipótese de que o risco de DRT seria maior nos negros e mulatos foi ainda influenciada pelos resultados de diversos levantamentos feitos na população geral, que têm mostrado de forma consistente maior incidência de DRT em pessoas negras do que em brancas 2,16-19.

É também importante observar que, diferente dos trabalhos anteriores, no presente estudo a população alvo foi de pacientes com glomerulonefrites primárias e não a população geral. Mesmo que as estimativas das associações entre raça e DRT na amostra selecionada reflitam corretamente o que ocorre em pacientes acompanhados no serviço onde o estudo foi realizado, é possível que, na população do estado da Bahia, a incidência de DRT secundária às glomerulonefrites seja maior em negros e mulatos do que os brancos. Isso pode ocorrer, por exemplo, se a prevalência de glomerulonefrites na população da Bahia for maior em negros e mulatos do que em brancos. Infelizmente não existe nenhum estudo que permita estimar com segurança as diferenças raciais na prevalência de glomerulonefrites na população da Bahia. Além do mais, diferenças no grau de miscigenação racial entre os Estados Unidos e o Brasil (particularmente no estado da Bahia) devem ser consideradas.

De acordo com os resultados apresentados, a associação entre raça e incidência de DRT foi significantemente modificada pela presença de HA. No grupo sem HA, a associação entre raça e incidência de DRT seguiu o padrão geral da amostra, ou seja, os negros apresentaram um risco menor de DRT do que os brancos, mesmo após se levar em consideração a idade e o sexo dos pacientes. Foi também avaliada a influência do tipo histológico na associação entre raça e incidência de DRT. O ajuste para tipo histológico foi feito devido a evidência de uma maior freqüência de EGF em negros ${ }^{7,8}$. Quanto a possíveis diferenças na sobrevida renal entre os tipos histológicos, os estudos publicados não permitem uma conclusão mais definitiva. Em uma revisão da literatura feita através do MEDLINE foi possível cons- tatar a falta de estudos visando comparar o prognóstico de pacientes com EGF e GNMP, os tipos histológicos mais freqüentes entre os pacientes biopsiados no HUPES. Tem sido mostrado, no entanto, que a sobrevida renal em pacientes com GNMP e EGF varia de forma importante na dependência de diversos fatores, incluindo a presença hipertensão arterial ${ }^{20,21}$. No presente trabatho, conforme esperado, GNMP foi o tipo histológico mais freqüente em brancos contrastando com a maior freqüência de EGF em negros. A incidência de DRT foi significantemente maior em pacientes com GNMP do que em portadores de EGF, apenas no grupo de normotensos. Estes achados explicam porque a associação entre raça e DRT em pacientes sem HA se tornou menos intensa após o ajuste para o tipo histológico.

No grupo de pacientes hipertensos, a associação entre raça e DRT apresentou uma direção contrária à observada entre os pacientes normotensos. Embora o tamanho relativamente pequeno da amostra torne os resultados vulneráveis à influência do acaso, a modificação de efeito exercida pela hipertensão arterial sobre a associação entre raça e DRT, merece ser melhor explorada em estudos futuros. É possível que negros e mulatos (comparados com brancos) portadores de glomerulonefrites primárias apresentem uma maior susceptibilidade para degradação da função renal apenas na presença de hipertensão arterial. Esta hipótese é consistente com a pior evolução da função renal em negros do que em brancos portadores de hipertensão arterial primária ${ }^{3}$.

Apesar do presente trabalho trazer achados relevantes, não se pode negar as limitações metodológicas inerentes a uma análise retrospectiva de dados colhidos de prontuários médicos. É importante observar, no entanto, que, no que se refere a classificação da variável raça, um estudo 
anterior mostrou que pacientes do Estado da Bahia classificados no prontuário médico como negros e mulatos apresentam uma forte associação com sobrenome de conotação religiosa ${ }^{22}$, uma característica relacionada com ancestralidade africana ${ }^{23}$. Esta associação foi similar a que tem sido relatada em estudos em que a classificação de raça foi feita pelo próprio investigador, seguindo critérios pré-definidos ${ }^{24}$. Embora não se possa afastar em definitivo a possibilidade de erro no diagnóstico de $\mathrm{HA}$, acredita-se que o uso da média de três determinações de PA tenha reduzido este potencial problema. A definição de DRT tomando por base a necessidade de terapia de substituição da função renal é o critério que tem sido utilizado em diversos trabalhos. Esta informação, no entanto, pode faltar se por alguma razão o paciente com DRT não retorna ao Serviço de Nefrologia e falece sem iniciar terapia de substituição da função renal. A influência da perda de acompanhamento nos resultados do presente estudo não pode ser definitivamente afastada. Um outro ponto que pode ser questionado é que a avaliação de doenças sistêmicas que causam glomerulonefrites não seguiu um protocolo pré-definido. Em verdade, os procedimentos e estratégias para investigação de formas secundárias de glomerulonefrites variou de acordo com as características dos pacientes e a forma de apresentação da doença. É importante observar, no entanto, que todos os pacientes foram acompanhados por um período maior do que seis meses o que deu oportunidade de se observar manifestações de doenças secundárias não detectadas inicialmente.

Em conclusão, os dados do presente estudo sugerem que HA é um fator modulador da associação entre raça e incidência de DRT em pacientes com glomerulonefrites provenientes do estado da Bahia. A maior incidência de DRT em brancos normotensos do que em negros normotensos com glomerulonefrites, apesar de ter sido uma associação estatisticamente significante, deve ser avaliada em investigações futuras, de forma a permitir conclusões mais definitivas. Os resultados apresentados também sugerem que a associação entre raça e incidência de DRT é influenciada pelo tipo histológico da glomerulonefrite. Neste grupo de pacientes da Bahia, EGF, o tipo histológico mais prevalente no negro, apresentou uma incidência de DRT menor do que a GNMP. Se forem devidamente consideradas as limitações de um estudo retrospectivo, os achados apresentados deverão servir de base para o desenvolvimento de estudos prospectivos, desenhados de modo a explorar, com maiores detalhes, a influência da raça na incidência de DRT.

\section{SUMMARY}

\section{Association Between Race and In- cidence of End-Stage Renal Disease Secondary to Glomerulonephritis: IN- FLUENCE OF THE Histologic TYPE AND Presence of Arterial Hypertension}

ObJeCtive. To assess, retrospectively, the association between race and incidence of end-stage renal disease (ESRD) in patients with glomerulonephritis in a University Hospital of Bahia, Brazil.

METHOds. The sample was comprised of 79 patients with focal segmental glomerulosclerosis (FSG), 50 with membranoproliferative glomerulonephritis (MPGN) and 49 with other types of glomerulonephritis (OTGN), followed-up between 1970 and 1996 for at least 6 months. It was not included patients with acute glomerulonephritis, minimal change disease, crescentic glomerulonephritis, connective tissue diseases or patients with serum creatinine level greater than or equal to $4.0 \mathrm{mg} / \mathrm{dl}$. The patients were classified according to race as caucasian (white), mulatto or negro. For patients older than $18 \mathrm{yr}$, not taking antihypertensives drugs, hypertension was defined as a mean of three blood pressure measurements greater than or equal to 140 $\mathrm{mmHg}$ for systolic or $90 \mathrm{mmHg}$ for diastolic; for ages $\leq 18$ years. The definition was based on criteria recommended by the Task Force on Blood Pressure in Children.

RESULTS. In the normotensive group, the incidence of ESRD was $72 \%$ lower in mulattos and negroes (relative risk $(R R)=0.28$; 95\% confidence interval $(C I)=0.11-0.67)$. In the group with hypertension, the direction of association between race and ESRD was opposite to that observed in the normotensive group $(R R=1.49 ; 95 \% C l=0.68-4.34)$. The modification in the RR depending on the presence or the absence of hypertension was statistically significant $(p=0.01)$. In the normotensive group the RR shifted toward the null hypothesis after the inclusion of histologic type in the Cox model.

Conclusions. Among normotensive patients with glomerulonephritis the incidence of ESRD was greater in caucasians (whites) than in negroes or mulattoes. By contrast among hypertensive patients there was a trend for a higher risk of ESRD in negroes. The study suggests that hypertension and the histologic type of glomerulonephritis influence the association between race and ESRD.

Key Words: Race. Negro. End-Stage Renal Disease. Glomerulonephritis.

\section{ReferênCias Biblográficas}

I. United States Renal Data System 1998 annual data report. The National Institute of Health, the National Institute of Diabetes and Digestive and Kidney Diseases, Bethesda, MD, 1998.

2. Lopes AA, Port FK, James SA, Agodoa L. The excess risk of treated end-stage renal disease in blacks in the United States. J Am Soc Nephrol 1993; 3:1961-71.

3. Rostand SG, Brown G, Kirk KA, Rutsky EA, Dustan HP. Renal insufficiency in treated essential hypertension. N Engl J Med 1989; 320:684-8.

4. Martinelli R, Lopes AA, Silveira MAS, Rocha H. Distribution of primary glomerular diseases in Bahia, Brazil: twenty-five year experience. 
Nephrology 1997 (suppl I):SI 08.

5. Queiroz FP, Brito E, Martinelli R. Influence of regional factors in the distribution of the histologic patterns of glomerulopathies in the nephrotic syndrome. Nephron 1975; 14:466-70.

6. Simon P, Ramée MP, Autuly V, et al. Epidemiology of primary glomerular diseases in a French region. Variations according to period and age. Kidney Int 1994; 46: I $192-8$.

7. Lopes AA, Martinelli RP, Silveira MA, Rocha H. Diferenças raciais entre pacientes com Esclerose Glomerular Focal e Glomerulonefrite Membranoproliferativa residentes no estado da Bahia. Rev Ass Med Bras 1999; 45: I I 5-20.

8. Bakir AA, Bazilinski NG, Rhee HL, Ainis $\mathrm{H}$, Dunea $G$. Focal segmental glomerulosclerosis. A common entity in nephrotic black adults. Arch Intern Med 1989; 149: 1802-4.

9. Krieger H, Morton NE, Mi MP, Azevêdo ES, Freire-Maia N, Yasuda N. Racial admixture in northeastern Brazil. 1965; 29: I 13-125. Ann Hum Genet 1965; 29: I 13-25.

10. Task Force on Blood Pressure Control in Children. Report of the Second Task Force on Blood Pressure Control in Children. National Heart, Lung, and Blood Institute, Bethesda, Maryland. Pediatrics 1987; 79: I-25.

I I. Kleinbaum DG, Kupper LL, Morgenstern H. Epidemiology Research: Principles and Quantitative Methods. Belmont, CA: Lifetime Lear- ning Publications, 1982

12. Gahlinger PM, Abramson JH. Computer Programs for Epidemiologic Analysis: PEPI. Vol. USD Inc. Stone Mountain, GA, 1995.

13. Norusis MJ. SPSSforWindows: Base system user's guide, release 6.0. Chicago, IL: SPPS Inc, 1993.

14. Norusis MJ. SPSS for Windows: Advanced Statistics, release 6.0. Chicago, IL: SPSS Inc, 1993

I5. Luft FC, Fineberg NS, Miller JZ, Rankin LI, Grim CE, Weinberger MH. The effects of age, race and heredity on glomerular filtration rate following volume expansion and contraction in normal man. Am J Med Sci 1980; 279: I 5-24.

16. Lopes AA, Hornbuckle K, James SA, Port FK. The joint effects of race and age on the risk of hypertensive end-stage renal disease. Am J Kidney Dis 1994; 4:554-60.

17. Rostand SG, Kirk KA, Rutsky EA, Pate BA. Racial differences in the incidence of treatment for end- stage renal disease. N Engl J Med 1982; 306: | 276-9.

18. Lopes AA, Port FK. Differences in the patterns of age-specific black/white comparisons between end-stage renal disease attributed and not attributed to diabetes. Am J Kidney Dis 1995; 25:7|4-2|.

19. Lopes AA. The joint effects of race and age on the risk of end-stage renal disease. Department of Epidemiology, The University of Mi- chigan (Doctoral Thesis), 1994

20. D'Amico G. Influence of clinical and histological features on actuarial renal survival in adult patients with idiopathic IgA nephropathy, membranous nephropathy, and membranoproliferative glomerulonephritis: survey of the recent literature. Am J Kidney Dis 1992; 20:3 I 5-23.

21. Rydel JJ, Korbet SM, Borok RZ, Schwartz MM. Focal segmental glomerular sclerosis in adults: presentation, course, and response to treatment. Am J Kidney Dis 1995; 25:534-42.

22. Lopes AA. Raça e Incidência de Doença Renal Terminal em Pacientes com Glomerulonefrites Primárias. Departamento de Medicina, Universidade Federal da Bahia (Tese de Livre Docência), 1997.

23. Azevêdo ES. The anthropological and cultural meaning of family names in Bahia, Brazil. Current Anthropol 1980; 21:360-63.

24. Azevêdo ES, Pinto da Costa TP, Silva MCBO, Ribeiro LR. The use of surnames for interpreting gene frequency distribution and past racial admixture. Hum Biol 1983; 55:235-42.

Artigo recebido: 30/06/1999

Aceito para publicação: 27/04/2000

\section{IMAGEM EM MEDICINA}

Participe da nova seção da Ramb. É um espaço aberto ao leitor, que poderá enviar material de interesse educativo, como fotos, ilustrações e exames, acrescido de três linhas explicativas contendo ainda nome do autor e serviço onde foi realizado. 0 material poderá ser enviado para a

Rua São Carlos do Pinhal, 324 - Cep 01333-903

S. Paulo - SP - E-mail: ramb@amb.org.br

NR: D. Fedrizzi integra o quadro de autores do artigo "Prevalência de nódulos de tireóide detectados por ecografia em mulheres após os 40 anos" (Ramb volume 46(4) 2000, página 331) 